\title{
ORIENTACIÓN AL MERCADO DE LAS EMPRESAS EXPORTADORAS AGROINDUSTRIALES DE LAS CIUDADES DE PEREIRA Y MANIZALES ${ }^{1}$
}

\section{Diana Patricia Jaramillo Parra ${ }^{2}$ \\ Luz Adriana Tabares Giraldo ${ }^{3}$}

Para citar este artículo: Jaramillo, D. y Tabares, L. (2015). “Orientación al mercado de las empresas exportadoras agroindustriales de las ciudades de Pereira y Manizales”. Inquietud Empresarial. Vol. XV (1), pp.141-161

Fecha de recepción: 27 de noviembre de 2014 Fecha de aceptación: 09 de marzo de 2015

\footnotetext{
1 Artículo de investigación resultado del trabajo de grado de maestría titulado "Orientación al mercadeo de las Empresas exportadoras de las Ciudades de Manizales y Pereira" otorgado por la Universidad de Manizales.

2 Ingeniera Agrónoma, Magíster en Mercadeo de la Universidad de Manizales - Colombia. cuantica82@gmail.com

3 Administradora de Empresas, Magíster en Mercadeo de la Universidad de Manizales - Colombia. adriatabag@hotmail.com
} 


\title{
RESUMEN
}

Este escrito describe la orientación al mercado de las empresas agroindustriales exportadoras a partir de las prácticas y actividades orientadas a los mercados internacionales y cómo permanecen en el tiempo, generando así ventajas competitivas frente a otras organizaciones. La investigación se orientó según los modelos de Naver y Slater y Kohly y Jaworski. La investigación realizada es de carácter descriptivo y teniendo en cuenta el manejo informal de las prácticas y actividades empresariales se optó por un estudio de tipo cualitativo, mediante entrevista a profundidad realizada a seis empresas agroindustriales exportadoras ubicadas en las ciudades objeto de estudio. Los resultados de la investigación permitieron determinar que de los componentes que forman parte de la orientación al mercado, la orientación al cliente es la línea principal para los empresarios.

\section{PALABRAS CLAVE}

Cliente, competencia, interfuncionalidad, capacidad de respuesta

\section{Market orientation of export firms agroindustriales cities of Pereira and Manizales}

\begin{abstract}
This paper describes how market orientation from practices and activities undertaken by the exporting agribusiness companies reach international markets and remain over time, generating competitive advantages over other organizations. The aim was to identify the practices and activities of agribusiness exporters of Manizales and Pereira market oriented along the lines of the model Naver and Slater and Kohly and Jaworski. The research conducted is descriptive and considering the informal management practices and business activities chose a qualitative study, using depth interviews conducted six exporting agribusinesses in the cities studied. The research results have revealed that the components that are part of market orientation, customer orientation is the main line for entrepreneurs.
\end{abstract}




\section{KEY WORDS}

Customer, competition, interoperability, responsiveness

\section{Orientação ao mercdo das empresas exportadoras agroindustriais das cidades de Pereira e Manizales}

\section{RESUMO}

Este escrito descreve a orientação ao mercado das empresas agroindustriais exportadoras a partir das práticas e atividades orientadas aos mercados internacionais e como permanecem no tempo, gerando assim vantagens competitivas frente a outras organizações. A investigação se orientou segundo os modelos de Naver e Slater e Kohly e Jaworski. A investigação realizada é de caráter descritivo e tendo em conta o manejo informal das práticas e atividades empresariais se optou por um estudo do tipo qualitativo, mediante entrevista a profundidade realizada a seis empresas agroindustriais exportadoras localizadas nas cidades objeto de estudo. Os resultados da investigação permitiram determinar que dos componentes que formam parte da orientação ao mercado, a orientação ao cliente é a linha principal para os empresários.

\section{PALAVRAS CHAVE}

Cliente, competência, interfuncionalidade, capacidade de resposta.

\section{INTRODUCCIÓN}

La región del Eje Cafetero, es la segunda en importancia económica del país, representa el 17,6\% del PIB de Colombia, participa con cerca del $16 \%$ de su base empresarial, y genera $12,7 \%$ de las exportaciones totales. En 2011, las exportaciones no tradicionales realizadas desde la región, alcanzaron los USD 6.273 millones, y registraron un crecimiento promedio anual de $12 \%$ en los últimos 5 años. Si bien los productos básicos como oro, café y banano representan un poco más del $50 \%$ del total exportado, la región tiene una oferta diversificada de bienes de mayor valor agregado entre los que se destacan productos alimenticios (Confecamaras, 2012).

Aunque el sector agroindustrial señala que las exportaciones muestran un crecimiento, la participación de las exportaciones de Caldas es escasa. De 
acuerdo con un estudio realizado por la Confederación Colombiana de Cámaras de Comercio, las ventas al exterior representa 1,1\% mientras que en Risaralda constituye el $1.0 \%$ (Confecamaras, 2012). Estas cifras se complementan con el informe de exportaciones reportado por la Cámara de Comercio de Manizales en el año 2011, donde sólo 80 empresas de todos los sectores en Caldas exportan sus productos a países como Estados Unidos, Venezuela, Alemania y Canadá, entre otros, con ventas de US 321 millones aproximadamente; de las empresas mencionadas anteriormente 40 empresas exportan productos agroindustriales con ventas de US 147 millones de dólares, donde el café y sus derivados son los productos de mayor representatividad en la región.

Con el objetivo de alcanzar una mayor participación en los mercados internacionales, se vienen implementando diversas estrategias en la región enfocadas en la especialización productiva; además de la identificación de apuestas productivas, incluyen temas de emprendimiento, innovación e inversión que buscan fortalecer e incrementar la base empresarial en aquellas actividades en las que la región presenta oportunidades significativas de cara a mercados internacionales.

Como requisito fundamental para esta especialización productiva es de vital importancia la identificación y construcción de actividades y prácticas orientadas al mercado que les permitan obtener ventajas significativas y así mismo pensar en un mayor aprovechamiento futuro de los tratados de libre comercio. Sin embargo, dicho aprovechamiento requeriría de acciones concretas en temas organizacionales y en procesos de innovación que se vienen desarrollando en su interior, por lo cual resulta importante conocer los aspectos teóricos más relevantes de la orientación al mercado con el fin de llegar a la identificación de estas prácticas y actividades que vienen realizando las empresas exportadoras agroindustriales en las ciudades de Manizales y Pereira.

\section{MATERIALES Y MÉTODOS}

La investigación realizada es de carácter descriptivo porque se orientó a identificar los comportamientos, prácticas y las actividades orientadas al mercado, realizadas en las tres empresas agroindustriales exportadoras de Manizales y tres empresas de Pereira con las mismas características. 
Por el carácter informal de las prácticas y actividades se decidió hacer un estudio de tipo cualitativo basado en los métodos planteados por Bonilla y Rodríguez (2005) con el fin de no intervenir en el carácter natural que caracteriza a este tipo de empresas y posibilitar que emergieran aspectos propios de su gestión cotidiana. Con la información obtenida se procedió hacer un análisis comparativo con los modelos de Kohli y Jasworski y Narver y Slater cuyos resultados sirvieron de base para estructurar los lineamientos para las compañias actuales y futuras que deseen orientarse al mercado.

El diagrama del proceso de investigación cualitativa planteada por Bonilla y tomado como diseño estratégico de la investigación abarca desde la definición de la situación y problema, el trabajo de campo, el análisis de la información, la comparación con el marco teórico y la identificación de componentes comunes que evidencian los resultados obtenidos.

Para la recolección de la información que permitió el acercamiento y análisis al objeto de estudio se utilizó la entrevista semiestructurada como herramienta para extraer la información de tipo cualitativo. En ella se tuvo en cuenta los componentes de las escalas de medición de orientación al mercado de los modelos de Naver y Slater y Kohli y Jaworski. La entrevista se llevó a cabo de manera individual a profundidad a las personas claves para el estudio que son gerentes y dueños de las empresas exportadoras y gerentes de mercadeo.

Se realizaron preguntas sobre experiencias y comportamientos así como las acciones y prácticas frente a situaciones plasmadas en la guía y lo que emergía en el proceso de la entrevista, permitiendo que los entrevistados se expresaran libremente y de forma particular sobre las formas y actuaciones con las que orientan sus empresas al mercado.

Los criterios de selección de la muestra se hicieron bajo el criterio de Patton (1990) quien manifiesta que todos los tipos de muestreo en la investigación cualitativa deberían encuadrarse bajo el rótulo general de muestreo intencional, el cual fue utilizado en la investigación, puesto que se determinó antes de empezar y los entrevistados se contactaron intencionalmente.

Las entrevistas fueron sistematizadas y analizadas en matrices, las cuales se estructuraron revisando el marco teórico hasta identificar los 
primeros componentes para el cumplimiento de los objetivos abordados en la investigación. Todos los análisis fueron hechos a partir de los datos obtenidos de la trascripción literal de las entrevistas, de los diarios de campo, de las conclusiones extraídas de las reuniones del equipo de investigación, de la revisión documental y de la reflexión intelectual de las empresas estudiadas y las corrientes teóricas basadas en los autores Naver y Slater y Kohli y Jaworski.

Los resultados obtenidos permitieron ir detectando diferencias y semejanzas, a partir de los cuales se realizaron matrices de análisis que a su vez sirvieron para la definición de los componentes descriptivos base de la investigación. Estos factores fueron claves para la estructuración puesto que se identificaron y agruparon por su incidencia y homogeneidad.

\section{RESULTADOS}

La orientación al mercado está ligada a la adopción del concepto de marketing como filosofía de negocio. En este sentido, la orientación al mercado fue definida como una cultura organizativa (Slater y Narver, 1994). Concretamente, un conjunto de valores y creencias que priorizan los intereses de los consumidores con el fin de desarrollar una empresa viable en el largo plazo. Por otro lado, la orientación como el conjunto de actividades, procesos y comportamientos derivados de la implantación del concepto de marketing, es el significado planteado por (Kohli y Jaworski, 1990).

Los componentes de análisis propuestos en los modelos del marco teórico son: orientación al cliente; orientación a la competencia; coordinación interfuncional- inteligencia de mercados; coordinación interfuncional diseminación de la información del mercado; coordinación interfuncional - capacidad de respuesta e innovación

\section{Orientación al cliente}

A continuación se presentan las actividades y prácticas con relación a la orientación al cliente de las empresas analizadas:

1.1. Relaciones a largo plazo basadas en confianza: De acuerdo con las entrevistas realizadas en el estudio, estas empresas tienen vínculos de confianza con sus clientes los cuales les permiten conocer las preferencias 
de los mismos y por tanto responder adecuadamente a sus necesidades y exigencias, un reflejo de ello es: "Se visitan los clientes actuales para establecer relaciones a largo plazo basados en la confianza, la atención personalizada es mucho mejor". Otro entrevistado aseguró lo siguiente "El valor agregado que se le da al cliente es la creación de un vínculo y establecimiento de una relación de confianza".

1.2 Capacidad de respuesta a la información sobre las necesidades, deseos y expectativas actuales y futuras de los clientes: Las empresas estudiadas proporcionan respuesta al contenido de la información sobre sus clientes, esto facilita la ejecución de acciones orientadas al desarrollo de bienes y servicios adaptados a las necesidades de los clientes. Algunas respuestas que respaldan esta acción se mencionan a continuación: "Los clientes son los que definen cómo va el producto, la textura, el color, sabor y calidad" y "En las visitas que se realizan a los clientes se trata de indagar acerca de sus necesidades actuales y futuras, para la empresa ofrecer productos que sean acordes a estas"

1.3 Identificación de segmentos de los clientes: Narver y Slater (1993), señalan que la orientación al cliente implica necesariamente, en primer lugar, la comprensión de los consumidores, con el objetivo de que la empresa esté en la capacidad de crear un valor sostenible para ellos, y en segundo lugar, la suficiente coordinación interfuncional entre los departamentos que permita a la firma ofrecer un valor superior a los clientes. Para el caso, se destacan la identificación y segmentación de los usuarios. Adicionalmente, este acercamiento permite implementar estrategias de desarrollo de productos "Son los clientes los que nos piden las modificaciones en los productos y nosotros las adoptamos y buscamos los medios para cumplirles".

Así mismo, los empresarios se esfuerzan por adquirir estándares de calidad que son extensivos a los clientes, teniendo en cuenta las siguientes afirmaciones "La estrategia es lograr certificaciones que le den seguridad al cliente respecto a la calidad del producto y de su proceso"; "Los clientes realizan visitas a la finca, con el fin de que conozcan de primera mano los parámetros de calidad con los que trabajamos, además de retroalimentarnos de sus necesidades" 
De este modo, la segmentación de consumidores implementada por las empresas agroindustriales les permitió identificar las necesidades específicas de sus clientes en el mercado objetivo, logrando diseñar de manera más eficaz la mezcla de marketing para satisfacerlas. También que estas empresas de tamaño mediano pudieron crecer más rápido cuando obtuvieron una posición sólida en sus segmentos especializados, debido a que crearon una oferta de bienes o servicios más afinada y con un precio apropiado para el público objetivo. Algunas de estas lograron seleccionar canales de distribución y de comunicación adecuados para su campo, e inclusive se enfrentaron a menos competidores.

1.4 Diseminación de la información para orientar a toda la organización hacia un objetivo en común "el cliente": se recopila la información de los clientes de manera informal, seguidamente se hace circular entre todos los niveles de decisión de la empresa, así: "Solo tomamos decisiones con respecto a los productos con la información que recolectamos de las visitas a los clientes y de las ferias". Posterior a eso, se presentan equipos de trabajo pequeños, pero que involucran a todas las áreas de la empresa "Hacemos reuniones permanentes en las que ponemos al tanto a todo el equipo de las necesidades de los clientes, para tomar decisiones en conjunto"

1.5 Servicio al cliente, medición de los niveles de satisfacción de los clientes: en las empresas estudiadas, existe un común denominador, el objetivo central de las operaciones se centra en la creación de un valor superior para los clientes. La organización trabaja para satisfacer las necesidades, deseos y expectativas de los clientes. En este sentido, la empresa analiza constantemente y de manera detallada los principales motivos de reclamaciones. "Se reciben las quejas de los clientes, y se analizan con el fin de tomar los correctivos necesarios".

Convierten la calidad y la satisfacción, (medidas desde la óptica de los clientes), en los valores más importantes en la cultura de la empresa. "Se mantiene una comunicación continua con el cliente para conocer como le llegó el producto y si cumplió con sus expectativas". Otra posición apunta al mejoramiento continuo "Yo, como gerente estoy al tanto de todos los procesos, visitando constantemente el cliente para recoger todas las recomendaciones". Adicionalmente, evalúan las etapas previas al proceso exportador "Antes de enviar el producto nos encargamos de evaluar detenidamente las muestras para estar seguros que el producto va a llegar 
con las condiciones transadas con el cliente". Otro informante asegura "Con frecuencia nos comunicamos con el cliente después de que el café ha llegado a su destino con el fin de conocer el grado de satisfacción del consumidor así como también saber si tienen alguna queja del producto o del proceso".

\section{Orientación a la competencia}

La orientación al mercado promueve la comprensión y gestión de información sobre los clientes de la empresa, competidores y fuerzas del entorno, de modo que pueda ser tratada colectivamente en la organización con la finalidad de crear y mantener una oferta que genere mayor valor. El objetivo final de la organización es reaccionar ante las necesidades del mercado y anticiparse a las mismas con una oferta más satisfactoria que sus competidores. Desde esta perspectiva, puede decirse que una empresa orientada al mercado es, en sí misma, una organización orientada al aprendizaje (Tuominen, Möller y Rajala, 1997), fortaleciéndose las creencias y comportamientos orientados al mercado por los principios propios del aprendizaje organizativo (Jaworski y Kohli, 1996). En este sentido, la importancia del aprendizaje según Day (1994) "constituye la única fuente de ventajas competitivas sostenibles"

2.1 Análisis de las estrategias de la competencia: la relación entre la orientación al mercado y el benchmarking se lleva a cabo indirectamente a través de la comparación de la empresa respecto a sus competidores tanto en los costos como las estrategias. Así la compañía, al tratar de igualar a la competencia en sus aspectos más destacados, gana en competitividad, satisfacción del cliente y finalmente en orientación al mercado.

Es importante entonces reconocer que el empleo de técnicas de benchmarking puede ayudar a lograr un mayor éxito en la implantación de una filosofía de orientación al mercado como se puede apreciar en las actividades que realizan las empresas estudiadas: "Conozco mi competencia y realizo un análisis constante de sus productos con respecto a los ofrecidos por mi empresa" "Estamos haciendo constantemente estudios acerca de los productos y servicios que ofrece la competencia con el fin de tener una idea más clara de nuestras ventajas competitivas o de lo que debemos mejorar". 
2.2 Desarrollo de ventajas competitivas que los diferencien en el mercado: en el caso de las empresas analizadas, el estar enfocadas a un segmento particular en el sector les ha permitido ajustar su cadena de valor a ese segmento teniendo como resultado costos más bajos en comparación con sus competidores. Uno de los informantes manifestó "El valor agregado que nosotros le brindamos a nuestros clientes es la innovación que nosotros aplicamos a nuestros productos ajustando el proceso de producción a las necesidades que encontramos con los clientes". De este modo, cada actividad de valor representa tecnología, conocimientos (know-how), procedimientos, acciones dentro del equipo de proceso. El conjunto de actuaciones empleadas por estas empresas es muy amplio, yendo desde obtener certificaciones de calidad para mejorar su producto e imagen hasta transportar bienes, lo que les ha permitido diferenciarse en su segmento especifico del mercado. Cabe anotar que el uso de tecnología no significa innovaciones radicales sino innovaciones incrementales puesto que se van dando de acuerdo con las especificaciones que va solicitando el cliente.

2.3 Capacidad de respuesta frente a las acciones de la competencia: las empresas desarrollan bienes y servicios adaptados a las necesidades de los clientes, según la siguiente respuesta "Realizamos estudios constantes sobre las certificaciones que nuestros clientes necesitan para ser más atractivos que la competencia"

Las empresas analizadas han obtenido ventajas competitivas, implementando estrategias basadas en las solicitudes del cliente, según ellas mejor que sus competidores. La ventaja competitiva depende no solo de comprender la cadena de valor de la empresa, sino de cómo encaja la empresa en la satisfacción de las necesidades del cliente. Estas empresas del sector agroindustrial difieren entre sí en cuanto a su historia y estrategias pero tienen como punto afín el cliente y el mercado.

\section{Coordinación Interfuncional}

Hace referencia a la utilización coordinada de los recursos de la compañía para crear un valor superior para los clientes, para Narver y Slater (1990), la coordinación interfuncional se centra en las actividades que incrementan la integración inter/intrafuncional y la cooperación, además de la generación de inteligencia de mercado, la diseminación (comunicación) y la respuesta de toda la organización. Sin embargo ambos trabajos destacan 
el componente comportamental en la orientación al mercado y, como dicen Kumar, Subramanian y Yauger (1997) "ambas perspectivas operacionalizan el constructo de orientación al mercado como un concepto unidimensional, en el que únicamente la medida de las dimensiones provoca diferentes resultados".

Como se mencionó en párrafos anteriores, el uso de la información del mercado tiene como origen el contacto directo con los clientes, quienes describen la experiencia de compra y la utilización de los productos.

En cuanto a la trayectoria y práctica en el mercado, se encontró que el desempeño de la empresa se encuentra determinado por la experiencia que obtiene de desempeños anteriores. Estas organizaciones tenderán a una actitud más favorable hacia algunos recursos si éstos supusieron una ventaja para la organización en años anteriores, en otras palabras, repetir lo positivo y evitar lo negativo (Gounaris y Avionitis, 1997). Al respecto, un informante manifestó "Las actuaciones están basadas en la experiencia que hemos adquirido, buscando siempre los mejores resultados de los procesos".

Referente a la investigación informal del mercado, algunas de las empresas exportadoras realizan investigación del mercado de una manera voluble puesto que los datos y la información que obtienen no la recopilan y archivan adecuadamente. Un empresario manifestó "Se utiliza la información que se ve en el mercado actual y las tendencias que se logran armar en ruedas y ferias de negocio y en lo que manifiestan los compradores y los consumidores". La generación de la inteligencia tiene que ver no sólo con las necesidades actuales, sino también con las futuras, lo que apremia a las organizaciones a anticiparse a las necesidades de sus consumidores, y a iniciar el camino para encontrarlas (Houston, 1986). Esta actividad la ejercen todas las áreas y los miembros de la empresa, pues como se mencionó inicialmente existe un sistema adecuado de comunicación, acompañado de una constante interacción. La información proviene de fuentes primarias y secundarias. La totalidad de empresas manifestaron la utilización de información procedente de fuentes institucionales como PROEXPORT, así como de las ruedas y ferias de negocios donde realizan contactos con clientes internacionales. 


\section{Diseminación interna de la inteligencia de mercado.}

Se refiere a la forma como las organizaciones comparten la información con todos los integrantes e intentan la comprensión y apropiación de la misma, con el fin de brindar elementos a cada área que apunten al crecimiento a partir de la información obtenida, estimulando interacciones formales e informales dentro de sus miembros.

La manera como las empresas exportadoras distribuyen la información, es en la mayoría de casos bajo un esquema informal y directo, es decir por medio del diálogo, ordenes de los superiores y en reuniones con el área administrativa y operativa

Lo anterior contribuye no sólo al crecimiento de las fuentes de información, sino también a una mayor fluidez de circulación. Para estas organizaciones el valor de la información generada sólo es maximizado cuando interrelaciona a todos los miembros de la empresa con el fin de facilitar su acción de respuesta, así lo revela la siguiente afirmación "Como es una empresa pequeña la información se comparte de manera directa a cada empleado, se manejan relaciones duraderas y los empleados que tengo llevan mucho tiempo trabajando conmigo, hay confianza para decirles las cosas"

Para las empresas la comunicación es fundamental y está ligada con la motivación y la confianza en los empleados. En el estudio se evidenció que las empresas con pocos departamentos y empleados tienden a comunicarse mejor y más rápidamente. Se genera un ambiente de confianza para que los empleados se comuniquen a tiempo, de esta manera circula información importante por todas las áreas de la empresa.

En la mayoría de casos los gerentes o el gerente de mercadeo son los encargados de convertir los datos en información relevante para la empresa. Esta función recae solo en ellos, pues la toma de decisiones está centralizada y por ende la responsabilidad es asumida por estos directivos.

\section{Diseño e implementación de una acción de respuesta.}

Esta respuesta se efectúa mediante estrategias y diseños de ofertas que se ajusten a los deseos y necesidades de los consumidores, con acciones concretas de la empresa que respondan a las estrategias implementadas 
por la competencia y adaptaciones de elementos de los factores del entorno tanto ambientales, sociales y jurídicos entre otros para favorecer la estrategia de la compañía. La implantación de esta respuesta supone la creación de un valor superior para el bien o servicio de la organización lo que crea una ventaja competitiva duradera.

Este elemento juega un papel importante en la orientación al mercado, puesto que la implementación de una acción de respuesta es el componente que genera mayor valor a las empresas y le suministra la oportunidad de un acercamiento al cliente, adaptarse al mercado y al medio en el que se desenvuelve.

Algunas de las respuestas en torno a este punto son "Si llegan especificaciones nuevas de los clientes toda la empresa se orienta a cumplirlas" "En el momento en que necesitamos mejorar un producto o diferenciarnos de la competencia, necesitamos la colaboración de todos los integrantes de la empresa".

\section{Innovación}

La identificación de los requerimientos y necesidades de los clientes es una de las fuentes principales de la innovación para las empresas analizadas. La innovación que se presenta en las empresas orientadas al mercado se da de manera incremental, es decir, las innovaciones no son de ruptura puesto que las empresas analizan constantemente el mercado y van realizando los cambios paulatinos que este le va pidiendo. Algunas respuestas brindadas por los directivos fueron "Se ha cambiado la planta varias veces para aumentar la capacidad de producción, esto se ha realizado con base en el crecimiento del mercado, se han cambiado proveedores de empaques para cumplir con las especificaciones de los clientes en ese sentido" "Se realizó una innovación en producto en bebedero, como la transformamos hacemos más eficiente el trabajo en el galpón porque no se pierda tanta agua y se mantiene más higiénico"

La generación de ideas que permitan satisfacer mejor a los clientes es parte de las obligaciones de todos los directivos y empleados. En las empresas analizadas se presenta una participación dinámica que permite una comunicación constante y eficiente como se observa a continuación: "Escucho constantemente las observaciones de los empleados acerca del producto o del proceso" "No, tenemos una estrategia definida hacemos 
reuniones periódicas, buscamos el aporte de la gente, su participación pero no tenemos definido actualmente".

Según lo anterior, los procesos de innovación interna que se generan en las empresas exportadoras carecen de una planeación o transferencia de conocimiento. Se hacen de manera informal y directa de los empleados hacia la gerencia y corresponde a innovaciones incrementales y no radicales.

\section{DISCUSIÓN DE RESULTADOS}

En las últimas dos décadas del siglo XX, se hicieron innumerables trabajos sobre la orientación al mercado, los que fueron realizados con el propósito de entregar una definición sobre el término y así determinar una medida para que sea de general aceptación y uso. Muchos de estos estudios guardan una estrecha relación en cuanto al tratar de generar un concepto que defina de alguna manera "la orientación al mercado" (Desphandé et al. 1993; Narver y Slater 1990, Slater y Narver 1994; Cadogan y Diamantopoulus, 1995; Jaworski y Kohli, 1993; Kohli et al., 1993). Se destaca la interacción de factores tanto externos: clientes, competencia, como internos: cliente interno, de la organización los cuales se constituyen en los elementos dinámicos en la orientación al mercado de las empresas.

Sin embargo se acentúan dos corrientes de pensamiento sumamente importantes, la orientación al mercado como filosofía (Narver y Slater, 1990) y la orientación al mercado como comportamiento (Kolhi y Jaworski, 1990). En primer lugar, Narver y Slater (1990), son los primeros en establecer una escala de medición de la orientación al mercado desde el punto de vista actitudinal, poniendo el acento en el carácter de competitividad y en la generación de una ventaja competitiva sostenible. Sostienen los autores, que el deseo de crear un valor superior para los consumidores y lograr conducir el negocio con una ventaja competitiva sostenible se basa en crear y mantener una filosofía que conlleva a las conductas precisas. Y es justamente la orientación al mercado como cultura de organización la que más efectiva y eficientemente crea los comportamientos necesarios para la generación de ese valor superior, permitiendo además continuar con una rentabilidad superior para el negocio.

En el trabajo de (Kohli y Jawoski, 1990), se establece que una organización 
orientada al mercado será aquella en la que estos tres pilares se manifiestan de forma operativa; por tanto, es imprescindible trasladar la filosofía a la práctica a través del desarrollo de un conjunto de actuaciones para llegar a una efectiva orientación al mercado. Para conseguir la aplicación al marketing y se concrete en una auténtica orientación al mercado hace falta incorporarle al concepto un componente más operativo y no ideológico.

En este propósito de estructurar el componente operativo, diseñan un modelo que explique la orientación al mercado, basado en las consideraciones previas, es decir; la orientación al cliente, la integración de las funciones de la empresa y la orientación al beneficio. Sin embargo, el modelo presenta factores adicionales; se trata de los antecedentes o condicionantes para la orientación al mercado, de los factores que pueden moderar el efecto de la misma, así como los resultados que ésta genera, más conocidos como antecedentes, modificadores y consecuencias.

Al realizar un análisis detallado de los planteamientos de (Kohli y Jaworski, 1990) y de (Narver y Slater, 1990), se pone de manifiesto la existencia de bastantes puntos en común entre ambos enfoques, tal y como posteriormente admiten (Narver y Slater 1994). Comparando estos componentes con los aportados por (Kohli y Jaworski, 1990), se encuentran grandes similitudes.

Los tres componentes de orientación al mercado mencionados por (Naver y Slater, 1994) comprenden actividades de adquisición de información de mercado, de su diseminación y coordinación en la creación de valor para el cliente, que resultan formar parte de los pilares de la orientación al mercado, según (Kohli y Jaworski, 1990).

Así, la orientación al mercado y la orientación a la competencia incluyen actividades referentes a la adquisición de información sobre compradores y competidores en el mercado objetivo, y de su difusión por toda la empresa. Por otro lado, el tercer componente de coordinación inter-funcional comprende los esfuerzos coordinados que van más allá del departamento de marketing y que crean un valor superior para el cliente.

Por tanto, para completar la visión de la orientación al mercado es importante tener en cuenta una perspectiva comportamental u operativa que implica la definición del concepto señalando las actividades que deben ser ejecutadas por una organización. 
Algunos estudios que han considerado simultáneamente un enfoque cultural y operativo en su relación con el desempeño organizativo han encontrado congruencia entre ambas medidas de orientación al mercado (Diamantopoulos y Hart, 1993; Avlonitis y Gounaris, 1997; Álvarez et al., 2000; Homburg y Pflesser, 2000). De hecho, la congruencia entre ambas ha sido utilizada como argumento de validez convergente en la evaluación de diversas escalas operativas de orientación al mercado (Kohli et al., 1993; Deshpande y Farley, 1998a). Tales evidencias, llevan a asumir que la adopción cultural y la implantación operativa de la orientación al mercado están relacionadas en gran medida.

La orientación cultural al mercado puede considerarse un antecedente de la orientación operativa al mercado (Álvarez et al., 2000; Homburg y Pflesser, 2000). Narver y Slater (1998) señalan que, cuando se mide la orientación al mercado a través de determinadas actividades específicas, se está midiendo la manifestación del sistema subyacente de creencias. Por tanto, resulta lógico asumir que la orientación de las actividades y procesos de la organización hacia la satisfacción de las necesidades del consumidor, esté necesariamente basada en la creencia de que constituye el medio para garantizar una posición ventajosa en el largo plazo.

Otro argumento a favor de este planteamiento es el hecho de que el interés de la alta dirección constituya un determinante de la implantación de la orientación operativa al mercado (Jaworski y Kohli, 1993), (Selnes et al. 1996), (Bhuian, 1998). Sin embargo, el argumento opuesto también ha sido contemplado en la literatura (Griffiths y Grover, 1998). El comportamiento constituye la base para el desarrollo progresivo de las creencias, valores y actitudes que constituyen la cultura organizativa. Es más, el énfasis investigador en determinar las actividades que caracterizan a una empresa orientada al mercado, ha facilitado la adopción de 'recetas operativas' no siempre amparadas en una conversión cultural. Todo ello permite inferir que a pesar de la relación entre la orientación cultural y operativa al mercado, puedan coexistir organizaciones orientadas a una de las perspectivas consideradas.

La adopción de actividades y procesos orientados al mercado puede estar basada en una imposición corporativa, o en la intención de seguir las tendencias actuales de gestión, y no en la convicción de todos los departamentos. Esto implica que, aun cuando exista cierta relación entre 
la orientación cultural y operativa al mercado desde la perspectiva de la función de producción, la dimensión cultural no es necesariamente un antecedente o condición fundamental de la dimensión operativa.

En el caso de las empresas agroindustriales exportadoras se pudo observar que existe una relación entre las actitudes y valores de los directivos y las actividades y prácticas que se realizan en la empresa. Como apuntan Griffiths y Grover (1998), la causalidad puede ser bidireccional, debido a que, por un lado, el desarrollo de una cultura organizativa deriva en comportamientos acordes con ella, por otro lado, el comportamiento de la organización, la creación de normas y la implantación de prácticas constituye la base para la formación de creencias y valores dentro de la organización. Esto explica la existencia de empresas donde hay correspondencia entre el grado de perspectiva cultural de los directivos y el grado de perspectiva comportamental de la empresa en sus acciones de orientación al mercado.

Esta circunstancia resulta evidente al observar las expresiones de los informantes, muchas de ellas dan cuenta de que las actividades y prácticas que realizan tienen una orientación al mercado comportamental que no es necesariamente un antecedente de la orientación al mercado cultural, sino que existen empresas con prácticas orientadas al mercado aunque sus directivos no prioricen los valores de orientación al mercado. Podría decirse entonces que algunas empresas adoptan la orientación al mercado a modo de instintivo y no como filosofía de gestión.

De acuerdo con lo mencionado anteriormente se puede inferir que las empresas analizadas presentan una orientación al mercado comportamental por expresarse en sus prácticas y actividades y esta operativización constante hace que se forme directa e indirectamente una cultura orientada al mercado dentro de cada empresa.

Es importante hacer dos aclaraciones en este punto. Por un lado, cualquier referencia al grado de orientación al mercado se realiza en términos relativos. El grado de orientación al mercado cultural y comportamental percibido dentro de las empresas es elevado. Por tanto, cuando se considera orientada al mercado significa que su grado de orientación es alto teniendo en cuenta las actividades y prácticas que realizan, lo cual es el reflejo más importante y representativo de dichas empresas.

En las empresas agroindustriales se presenta una ausencia de sistemas 
de información confiables y oportunos, adicionalmente, no hacen uso de información obtenida a partir de investigaciones del mercado formales o contratadas a terceros.

Aunque refieren que los clientes se sienten satisfechos, no existen datos formales que lo demuestren. Esto puede convertirse en una debilidad en el largo plazo si se maneja la hipótesis de que el número de clientes aumenta, entonces la interacción directa con el cliente tiende a disminuir.

Otro elemento asociado a la capacidad de respuesta es el manejo de los tiempos, pues aunque se responde adecuadamente no se cuantifica y tampoco existe un protocolo que permita hacer seguimiento.

En general los modelos de Narver y Slater y Kohli y Jaworski tienen los componentes de orientación al mercado que desarrollan las empresas exportadoras pero su aplicación y reflejo en las actividades y prácticas se da de una manera informal.

\section{CONCLUSIONES}

El dinamismo que rodea el entorno de la empresa, necesariamente la obliga a adecuarse y a orientarse al mercado si quiere seguir siendo competitiva. Esta capacidad o habilidad de adaptarse a los cambios, es la esencia misma de la orientación al mercado, ya que esta puede dotar a la empresa de flexibilidad y dinamismo si es empleada oportuna y correctamente.

La orientación al mercado de las empresas agroindustriales exportadoras se evidencia en las diferentes actividades y prácticas que se realizan para conseguir los objetivos organizacionales.

De los componentes que forman parte de la orientación al mercado, es la orientación al cliente la que se observó como la línea principal. Fueron las prácticas y actividades desplegadas para obtener la diferenciación sobre la base del ofrecimiento de un valor superior al cliente, las que mayor relevancia adquirieron en el análisis de las empresas estudiadas. Esto se evidenció a través de nuevos productos totalmente adaptados a las necesidades y demandas de los consumidores.

Adicionalmente, carecen de una orientación al mercado enmarcada desde una cultura o filosofía de la organización, ésta surge de manera espontánea e informal marcada por el estilo de dirección del gerente y la función 
acción, es decir, primero vienen las acciones, la operativización de la orientación al mercado lo que se convierte con el tiempo en una cultura para la empresa la cual es liderada por el accionar del gerente.

\section{REFERENCIAS BIBLIOGRÁFICAS}

Álvarez, L., Santos. M. y Vásquez, R. (2000). Análisis cultural y operativo de la orientación al mercado: efectos moderadores en la relación OM Resultados. En: Revista Española de Investigación de Marketing ESIC, Vol. 4(1), pp. 7-41.

Avlonitis, G. y Gounaris, S. (1997). Marketing Orientation and Company Performance. Industrial vs Consumer Goods Companies. En: Revista Industrial Marketing Management, Vol. 26, pp.385-402.

Bhuian, S. (1998). An empirical examination of market orientation in saudi Arabian manufacturing companies. En: Journal of Business Research, Vol.43 (1), pp.13-25.

Bonilla, E., y Rodríguez, P. (2005). Más allá del dilema de los métodos. Colombia: Editorial Nomos S.A.

Cadogan, J. y Diamantopoulos, A. (1995). Narver and Slater, Kohli and Jaworski and the market orientation construct: integration and internationalization. En: Journal of strategic marketing, Vol.3, pp.41-60.

Confederación Colombiana de Cámara de Comercio (2012). Informe anual de exportaciones por departamentos. Recuperado 27/10/2014 de: http://www.confecamaras.org.co/phocadownload/ InformesGestion/informe_de_gestion_2011_2012.pdf.

Day, G. (1994). The Capabilities of Market-Driven Organizations. En: Journal of Marketing, Vol.58, pp.37-52.

Deshpande, R., Farley, J. y Webster, F. (1993). Corporate Culture, Customer Orientation and Innovative in Japanese Firms: A Quadrad Analysis. En: Journal of Marketing, Vol.57, pp.23-37. 
Deshpande, R. y Farley, J. (1998). Measuring Market Orientation: Generalization and Synthesis. En: Journal of Market Focused Management, Vol. 2, pp. 213-232.

Diamantopoulos, A. y Hart, S. (1993). Linking Market Orientation and Company Performance: Preliminary Evidence on Kohli and Jaworski's Framework. En: Journal of Strategic Marketing, No. 1, pp. 93-121.

Gounaris, S. y Avlonitis, G. (1997). Company and market correlates of marketing orientation. En: 25th EMAC Conference, Warwick. Proceedings, Vol. 3, pp. 536-555.

Griffiths, J. y Grover, R. (1998). A Framework for Understanding Market Orientation: The Behavior and the Culture. En: Marketing Theory and Applications, Vol. 9., pp. 311- 320.

Grewal, D., Pechmann, C. (Eds.), (1998). American Marketing Association Winter Educators' Conference Proceedings: Marketing Theory and Applications. 9. Chicago, Illinois: American Marketing Association, pp. $31-320$.

Homburg, Ch. y Pflesser, Ch. (2000). A Multiple-Layer Model of Marketoriented Organizational Culture: Measurement issues and Performance Outcomes. En: Journal of Marketing Research, Vol.37, pp. 449-462.

Houston, F. (1986). The Marketing Concept: What is and what is not. En: Journal of Marketing, Vol. 50 (2), pp. 81-87.

Kohli, A. y Jaworski, B. (1990). Market Orientation: The Construct, Research Propositions, and Managerial Implications. En: Journal of Marketing, Vol. 54, pp. 1-18.

Kohli, A. y Jaworski, B. (1993). Market Orientation: Antecedents and Consequences. En: Journal of Marketing, Vol. 57, pp. 53-70.

Kohli, A., Jaworski, B. y Kumar, A. (1993). MARKOR: A measure of Market Orientation. En: Journal of Marketing Research, Vol. 30(4), pp. 467- 477. 
Kumar, K., Subramanian, R. y Yauger, C. (1997). Performance-Oriented: Toward a Successful Strategy. En: Revista Marketing Health Serevices, summer, pp.10-20.

Narver, J., y Slater, S. (1990). The Effect of a Market Orientation on Business Profitability, En: Journal of Marketing, Vol 54, pp. 20-35.

Narver, J., y Slater, S. (1993). Market Orientation and Customer Service: the Implications For Business Performance, in E - European Advances in Consumer Research Volume 1, eds. W. Fred Van Raaij and Gary J. Bamossy, Provo, UT: Association for Consumer Research, pp. 317-321.

Narver, J. y Slater, S. (1994). Does Competitive Environment Moderate the Market Orientation-Performance Relationship. En: Journal of Marketing, Vol. 58, pp.46-65.

Narver, J. y Slater, S. (1998). Additional Thoughts on the Measurement of Market Orientation: A Comment in Deshpandé and Farley. En: Journal of Market Focused Management, Vol.2, pp. 233-236.

Patton, M. (1990). Qualitative evaluation and research methods. Beverly Hills, CA: Sage. Designing Qualitative Studies.

Slater, S. y Narver, J. (2000). Intelligence Generation and Superior Customer Value. En: Journal of the Academy of Marketing Science, Vol. 28(1), pp. 120- 127.

Selnes, F., Jworski, B. y Kohli, A. (1996). Market Orientation in United States and Scandinavian Companies. A Cross-Cultural Study. En: Scandinavian Journal of Management, Vol.12 (2), pp.139-157

Tuominen, M., Möller, K. y Rajala, A. (1997). Marketing Capability: A Nexus of Learning-Bases Resources and a Prerequisite for Market Orientation. $26^{\text {th }}$ EMAC Conference, Warwick, may, Proceedings, Vol. (1), pp.1220-1240. 